\title{
DEMON: a Proposal for a Satellite-Borne Experiment to study Dark Matter and Dark Energy
}

Alicia Berciano Alba ${ }^{a, b}$, Pedro Borges da $\operatorname{Silva}^{c}$, Hans Eichelberger ${ }^{d}$, Francesca Giovacchini ${ }^{e, f}$, Mareike Godolt ${ }^{g}$, Günther Hasinger ${ }^{h}$, Michael Lerchster ${ }^{i,} h$, Vincent Lusset ${ }^{j}$, Fabio Mattana ${ }^{k}$, Yannick Mellier ${ }^{l}$, Michał Michałowski ${ }^{m}$, Carlos Monteserin-Sanchez ${ }^{n}$, Fabio Noviello ${ }^{o}$, Carina Persson ${ }^{p}$, Andrea Santovincenzo ${ }^{q}$, Peter Schneider ${ }^{r}$, Ming Zhang ${ }^{s}$, Linda Östman ${ }^{t}$

${ }^{a}$ Joint Institute for VLBI in Europe, postbus 2, 7990 AA Dwingeloo, The Netherlands;

${ }^{b}$ Kapteyn Astronomical Institute, P.O.box 800, 9700AV Groningen, The Netherlands;

${ }^{c}$ Fundação Navegar, Centro Multimeios de Espinho, Av. $24 n^{\circ} 800$ Espinho, Portugal;

${ }^{d}$ Space Research Institute, Austrian Academy of Sciences, 8042 Graz, Austria;

${ }^{e}$ Department of Physics, Bologna University, via Irnerio 46, I-40126 Bologna, Italy;

${ }^{f}$ Istituto Nazionale di Fisica Nucleare, Viale B. Pichat 6/2, I-40127 Bologna, Italy;

${ }^{g}$ Astrophysical Institut Potsdam, An der Sternwarte 16, 14482 Potsdam, Germany;

${ }^{h}$ Max Planck Institute for Extraterrestrial Physics, Giessenbachstr., D-85748 Garching, Germany;

${ }^{i}$ University Observatory Munich, Faculty for Physics at the Ludwig Maximillians University, Scheinerstr. 1, 81679 Munich, Germany;

${ }^{j}$ Service de Physique des Particules, Commissariat á l'Energie Atomique - Saclay, bat 141

F-91191 Gif-sur-Yvette cedex, France;

${ }^{k}$ Instituto Nazionale di Astrofisica, Istituto di Astrofisica Spaziale e Fisica Cosmica di Milano, Via Bassini 15, 20133 Milano, Italy;

${ }^{l}$ Institut d'Astrophysique de Paris, 98 bis Boulevard Arago, F-75014 Paris, France;

${ }^{m}$ DARK, University of Copenhagen, Juliane Maries Vej 30, 2100 Copenhagen, Denmark;

${ }^{n}$ Instituto de Fisica de Cantabria, Avd. Los Castros s/n, E-39005 Santander, Spain;

${ }^{o}$ Dept. of Experimental Physics, NUI Maynooth, Maynooth, Co. Kildare, Ireland;

${ }^{p}$ Onsala Space Observatory, Chalmers University of Technology, S-439 92 Onsala, Sweden;

${ }^{q}$ ESA/ESTEC TEC-SYE, P.O. Box 299, NL-2200 AG Noordwijk ZH, The Netherlands;

${ }^{r}$ Argelander-Institut für Astronomie, Auf dem Hügel 71, D-53121 Bonn, Germany;

${ }^{s}$ University of Manchester, Jodrell Bank Observatory, Macclesfield SK11 9DL, UK;

${ }^{t}$ Department of Physics, Stockholm University, SE 106 91, Stockholm, Sweden;

\begin{abstract}
We outline a novel satellite mission concept, DEMON, aimed at advancing our comprehension of both dark matter and dark energy, taking full advantage of two complementary methods: weak lensing and the statistics of galaxy clusters. We intend to carry out a $5000 \mathrm{deg}^{2}$ combined IR, optical and X-ray survey with galaxies up to a redshift of $\mathrm{z} \sim 2$ in order to determine the shear correlation function. We will also find $\sim 100000$ galaxy clusters, making it the largest survey of this type to date. The DEMON spacecraft will comprise one IR/optical and eight X-ray telescopes, coupled to multiple cameras operating at different frequency bands. To a great extent, the technology employed has already been partially tested on ongoing missions, therefore ensuring improved reliability.
\end{abstract}

Keywords: observational cosmology, space missions, dark matter, dark energy

Further author information: (Send correspondence to A.B.A.)

A.B.A.: E-mail: berciano@astro.rug.nl, Telephone: +31 503633505

Space Telescopes and Instrumentation II: Ultraviolet to Gamma Ray, edited by

Martin J. L. Turner, Günther Hasinger, Proc. of SPIE Vol. 6266, 626633, (2006)

0277-786X/06/\$15 - doi: $10.1117 / 12.670660$

Proc. of SPIE Vol. 6266 626633-1 


\section{INTRODUCTION}

The open question concerning the nature of dark matter and dark energy is certainly one of the most compelling issues of modern astrophysics, that demands new and better observations in order to be answered. Until the physical properties of dark matter can be determined experimentally (something that cannot be done in the laboratory up to now), trying to improve our knowledge about these properties implies improving the current measurement of the cosmological parameters. In particular, in the case of dark energy, the first crucial step is to try to distinguish a cosmological constant model from time dependent quintessence models.

The current best constraints on the cosmological parameters come from the combined results of three different techniques with complementary degeneracies: SNIa experiments, CMB measurements, and the study of galaxy clusters. In addition to these well known methods, weak lensing is emerging as one of the most powerful and robust probes of the large scale structure of the universe and the nature of dark. ${ }^{1-3}$

Several existing and proposed wide-field weak lensing surveys, ground-based* and space borne ${ }^{\dagger}$ are currently being developed. In order to contribute to the design of possible space missions we present DEMON (Dark Energy and Matter Observational Nexus), a novel concept satellite conceived to study the properties of dark matter and dark energy combining two complementary techniques: weak lensing and clusters statistics.

\section{OVERVIEW: WEAK LENSING AND CLUSTER STATISTICS}

In order to address the above science questions, DEMON will carry out a combined optical/IR and X-ray widefield survey to provide the required data for weak lensing and cluster statistic studies. Sections 2.1 and 2.2 explain the basis of both techniques and the usefulness of combining them to study the problem of dark matter and dark energy.

\subsection{Weak lensing}

The weak gravitational lensing effect is the deflection of light by large scale structures along the line-of-sight. This produces a coherent distortion of the shape of observed background galaxies (cosmic shear), which contains information about the three dimensional mass distribution of the universe and also about the structure growth with time. The cosmic shear maps obtained from weak lensing surveys can be used to reconstruct the dark matter power spectrum, prove the effect of dark energy in the geometry of the universe (geometrical tests), and measure the cosmological parameters analysing the 3D shear power spectrum in redshift slices (tomography). These maps can be obtained through the statistical properties of the observed ellipticity of galaxies, which requires a large statistical sample of galaxies and very good image quality to measure the galaxy ellipticities reliably.

Since the cosmic shear is only due to gravity, weak lensing does not suffer from galaxy bias uncertainty like the baryon oscillation technique, and is not affected by evolution and/or environmental effects as may be the case for SNIa. In addition, weak lensing offers a unique method to probe the matter distribution in the transition domain from quasi-linear to non-linear scales at medium redshifts which cannot be addressed by any of the other techniques. Studies of cosmic shear already have led to significant constraints on cosmological parameters, most noticeably on the normalisation of the power spectrum. ${ }^{4}$

\subsection{Cluster statistics}

Clusters of galaxies are the largest and most recently collapsed objects in the universe. While their formation and evolution are driven by gravity, their large scale distribution and growth rate are also affected by dark energy. Two methods involving clusters are especially promising in cosmology: determining the redshift evolution of the matter power spectrum, and measuring the redshift evolution of the cluster mass function (the abundance of clusters as a function of mass). ${ }^{5}$ Comparing these statistical quantities with those predicted by structure formation theory allow us to measure the cosmological parameters and the equation of state of dark energy. ${ }^{6}$

\footnotetext{
*VISTA (Visible \& Infrared Survey Telescope for Astronomy), Pan-STARRS (Panoramic Survey Telescope And Rapid Response System), LSST (Large Synoptic Survey Telescope), CFHTLS (Canada-France-Hawaii Telescope Legacy Survey), KIDS (Kilo-Degree Survey) and DES (Dark Energy Survey)

${ }^{\dagger}$ JDEM (Joint Dark Energy Mission) from NASA-DOE and DUNE (Dark UNiverse Explorer) from CNES, also proposed for ESA's Cosmic Vision
} 
Cluster statistics require mass determination for each cluster, which can be inferred from observables such as the intracluster gas temperature in the X-ray spectrum, and the bolometric X-ray luminosity. ${ }^{7}$ Some Xray surveys of galaxy clusters have measured the power spectrum and the cluster mass function, ${ }^{8}$ but not its evolution with redshift. Missions like XMM-Newton with a random survey precludes any studies based on spatial correlation (such as the matter power spectrum). These effects are even more important for the Chandra X-ray Mission and the planned XEUS (X-ray Evolving Universe Spectroscopy Mission) and Constellation-X mission, with their smaller fields of view.

In order to reach estimates with errors of a few percent, observations of ten thousand galaxy clusters in contiguous fields and with well defined completeness are needed. ${ }^{5}$ This goal can be efficiently accomplished by a wide-field X-ray survey. In addition, the mass-luminosity/temperature relations needed for cluster statistics studies can be considerably improved with additional mass measurements ${ }^{9}$ provided by alternative methods like weak lensing. ${ }^{10}$ Since weak lensing and cluster statistics also provide complementary measurements of the cosmological parameters, it is very suitable to combine them in the same mission.

\section{SCIENCE CASE}

The main goal of the DEMON satellite is to provide optimum quality data for weak lensing studies, the scientific requirements are discussed further in Sect. 3.1. On the other hand, since cluster statistic studies are an interesting additional feature for the mission, we intend to take advantage of the X-ray telescopes developed for the eROSITA mission to carry out a simultaneous X-ray survey. The characteristics of such an X-ray survey are explained in Sect. 3.2.

\subsection{Weak lensing scientific requirements}

Since the weak lensing technique is based on measurements of galaxy shapes, high image quality is a mandatory requirement in order to achieve optimal results. That makes a space-based wide-field survey the natural option to overcome the limitations produced by the degradation of the images in ground based surveys (smearing due to atmospheric seeing, PSF instabilities, weather instabilities and telescope instabilities due to flexures and windshake). A space telescope provides a diffraction limited and stable point spread function (PSF) minimising the systematics of the images, and allowing the detection of more faint resolved galaxies.

In order to provide a high sample of galaxies to minimise statistical errors, we need a large sky coverage and a high number density of resolved galaxies for shape measurements. ${ }^{11}$ With a limiting magnitude of $\mathrm{I}_{\mathrm{AB}} \sim 27$, a galaxy density of $\sim 60-100 \mathrm{arcmin}^{-2}$ is expected. ${ }^{11-15}$ This provides a galaxy sample with $\langle z\rangle \sim 1.3-1.4,{ }^{13,16}$ suitable to probe the range ${ }^{17} z=0-2$ in which the effect of dark energy is more prominent. ${ }^{18}$ According to Tyson et al., ${ }^{11}$ ellipticity values can be retrieved for galaxies larger than $1.2 \times \mathrm{PSF}$. Since galaxies with $\mathrm{I}_{\mathrm{AB}} \sim 27$ have mean sizes $\left(2 \times\right.$ half-light radius) of $\sim 0.2^{\prime \prime},{ }^{14}$ we need a telescope with a full width half maximum (FWHM) of the PSF $\leq 0.17^{\prime \prime}$. In addition, accurate measurements of galaxy shapes require a FWHM sampling of at least $2 \times 2$ pixels for a proper PSF deconvolution. ${ }^{14}$

To assess the above scientific requirements, we studied a baseline model consisting of a 2 meter telescope similar to the one suggested for SNAP ${ }^{19}$ (SuperNova/Acceleration Probe). We intend to cover $5000 \mathrm{deg}^{2}$ with an exposure time of $4 \mathrm{ks}$ per filter. The instrumentation requirements are described in Sect. 4.1.

Weak lensing tomography also requires the determination of the photometric redshifts of the galaxy sample (too big for spectroscopic redshift measurements) which can be obtained with a targeted accuracy of the order of $0.03(1+z)$ using 8 filters. ${ }^{20}$ Since it is not practical to include them all in a single spacecraft, a groundbased optical wide-field imaging survey done in parallel will be needed to achieve the mentioned accuracy. The minimum requirement for the ground-based survey is a 4 meter diameter optical telescope with a $3 \mathrm{deg}^{2}$ field of view (FOV) operating in the U, B, V, and R bands. Projects of this kind, such as VISTA, KIDS, Pan-STARRS... are currently being developed. The IR filters should be included in the spacecraft, since the sensitivity of groundbased IR observations is severely limited by atmospheric and thermal effects. 


\subsection{X-ray survey characteristics}

The kind of X-ray survey that we can perform with DEMON is determined by: (1) the sky coverage $\left(5000 \mathrm{deg}^{2}\right)$ and exposure time (16 ks per pointing) imposed by the weak lensing mission requirements, and (2) the technical specifications of the X-ray telescopes developed for the eROSITA mission (see Sect. 4.2 for details).

The eROSITA telescopes are optimized for cluster statistics studies. In particular, they provide an angular resolution of $<15^{\prime \prime}$, suitable to distinguish between galaxy clusters and point-like X-ray sources. ${ }^{21}$ With an energy resolution of $130 \mathrm{eV}$ at $6 \mathrm{keV}$, they also provide an $\mathrm{X}$-ray spectrum with enough resolution to measure the mean cluster gas temperature.

An X-ray instrument composed by 8 of such a telescopes will have a total FOV of $3.55 \mathrm{deg}^{2}$. Combined with an exposure time of $\sim 64 \mathrm{ks}$ per pointing, such an instrument will reach a minimum flux of $8 \times 10^{-15} \mathrm{erg} \mathrm{cm}^{-2}$ $\mathrm{s}^{-1}$ in the energy range $0.5-2 \mathrm{keV}$. Under this conditions, the DEMON X-ray survey is expected to detect $\sim 20$ clusters per $\operatorname{deg}^{2}$ and identify, in the observed region of the sky, all the clusters with a mass $\gtrsim 2 \times 10^{14}$ $\mathrm{M}_{\odot}$ up to $z \sim 1.5$. Therefore, DEMON will produce a catalog of $\sim 100,000$ clusters with a mean redshift of 0.75. The flux-selection will avoid the contamination from clusters highly affected by non-gravitational heating, mainly non massive and non relaxed. ${ }^{8}$

The study of the evolution of the matter power spectrum and cluster mass function requires an error $\sim 0.02$ in the cluster redshift measurements. ${ }^{5}$ This information will be provided by the DEMON (combined with the required ground-based survey) with a median accuracy of 0.07 for the redshift of each galaxy. Therefore, redshift measurements of $\sim 12$ galaxies per cluster will be enough to achieve the required precision.

Another interesting feature of the DEMON compared to other X-ray surveys is that, due to the flux limit and survey area that is going to cover, it will explore a measurement domain that was not done before.

\section{INSTRUMENTATION}

The baseline optical concept will comprise a $2 \mathrm{~m}$ diameter optical/IR telescope encircled by eight $0.358 \mathrm{~m}$ diameter $\mathrm{X}$-ray telescopes. Below, the optical/IR and X-ray telescopes and detectors are described. The specifications of both instruments are summarized in Tables 1 and 2 .

\subsection{Optical/IR telescope and detectors}

The main DEMON optical/IR instrument is a three-mirror anastigmatic Korsch telescope ${ }^{22}$ (see Figure 1). This configuration produces an achromatic image limited by diffraction over a planar field of more than $1 \mathrm{deg}^{2}$, and has been used extensively in space. Honeycomb sandwich mirror technology is chosen to minimize the mass of the mirror.

DEMON will be equipped with 4 filters: one optical broad-band filter to measure the galaxy shapes $(\mathrm{R}+\mathrm{I}+\mathrm{z}$, centered at $0.8 \mu \mathrm{m}$ with $0.2 \mu \mathrm{m}$ width), and 3 IR filters to determine the photometric redshifts (J, H and K).

In the broad band filter, the size of the telescope FWHM PSF is $0.08^{\prime \prime}$, which requires an angular resolution of $0.04^{\prime \prime} /$ pixel (to be properly sampled) and a pixel size of $5 \mu \mathrm{m}$. Since this pixel size is much smaller than the current technology available, the DEMON pointing strategy will take advantage of micro-dithering ${ }^{23}-$ the integration time is divided into two sub-pointings separated by half a pixel. This allows us to use a pixel $\sqrt{2}$ times larger. Combined with a magnifying lens system (to enlarge the image by a factor of 1.5), the PSF can then be properly sampled using $10 \mu \mathrm{m}$ pixels. Since there is no need for this high resolution for the infrared filters, we can use IR detectors with the standard pixel size of $18 \mu \mathrm{m}\left(0.085^{\prime \prime} /\right.$ pixel $)$.

The DEMON focal plane is a ring with a partially vignetted area in the center, where the detectors are situated in four 20' $\times 40^{\prime}$ rectangular areas (see Figure 2). The four filters are settled in one direction to cover the same FOV, which requires an orthogonal scanning strategy. In total DEMON will have 128 CCDs $(2627 \times 2627$ pixels each $)$ for the broad-band filter, and 80 IR pixel arrays $(2335 \times 973$ pixels each $)$ for each of the IR filters.

The SNAP collaboration have successfully developed a new type of large format back-illuminated p-channel CCDs with $10.5 \mu \mathrm{m}$ pixels, specially suitable for space. ${ }^{24}$ Therefore, the development of $10 \mu \mathrm{m}$ pixels will not be a technical problem. For the IR detectors we can use $\mathrm{HgCdTe}$ devices from Rockwell with a $2.5 \mu \mathrm{m}$ wavelength cut-off. 


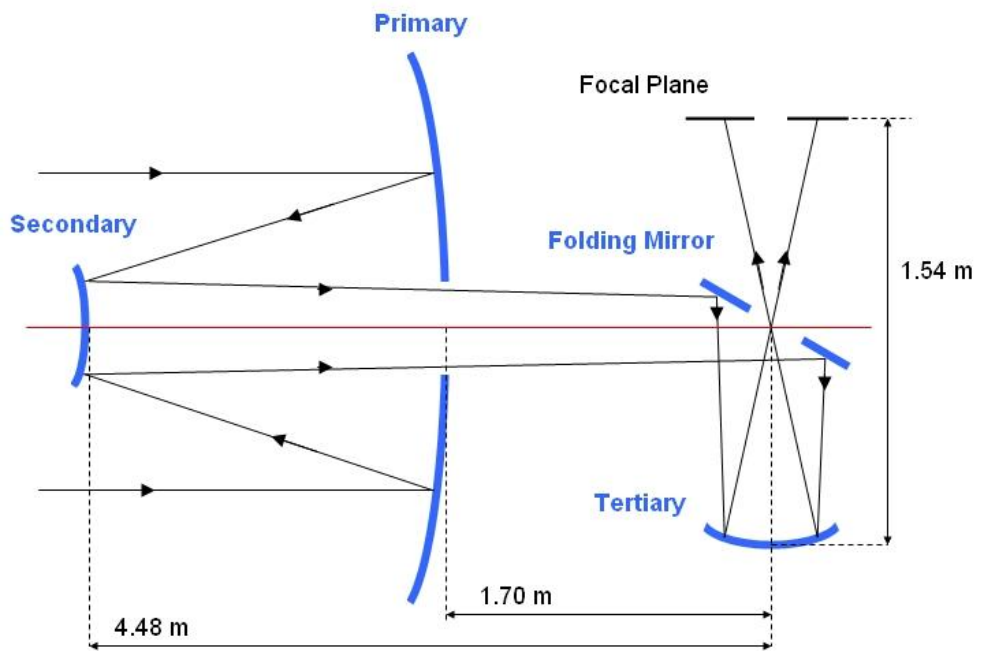

Figure 1. The optical mirror system (not to scale), without magnifying lens system.

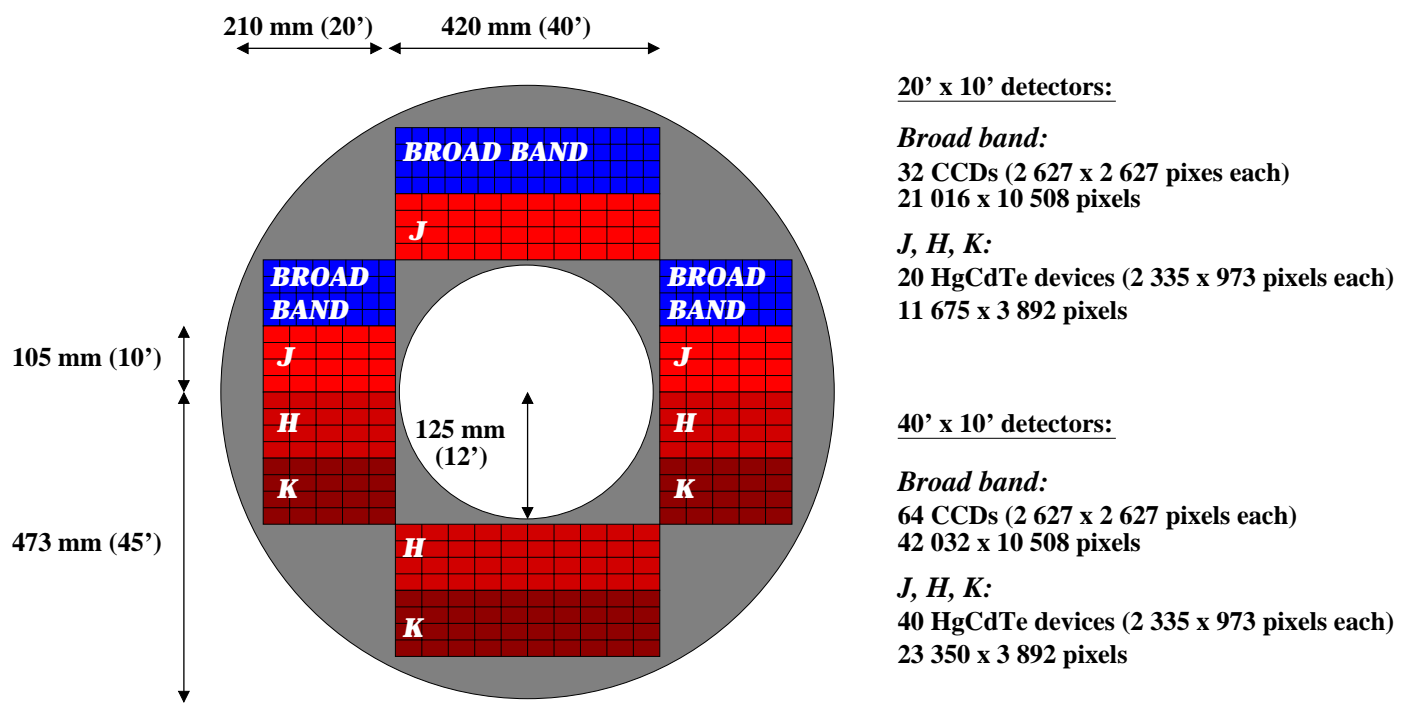

Figure 2. Optical/IR focal plane and detectors. 
Table 1. Specifications of the optical/IR system.

\begin{tabular}{ll}
\hline \hline Parameter & Value \\
\hline Telescope & 3 -mirror system \\
Focal length & $24 \mathrm{~m}$ \\
Effective focal length & $36.12 \mathrm{~m}$ \\
Primary mirror diameter & $2000 \mathrm{~mm}$ \\
Primary mirror thickness & $400 \mathrm{~mm}$ \\
Secondary mirror diameter & $466.7 \mathrm{~mm}$ \\
Tertiary mirror diameter & $1066.7 \mathrm{~mm}$ \\
Fold mirror diameter & $489.3 \mathrm{~mm}$ \\
Baffle length & $0.7 \mathrm{~m}$ \\
FOV & $0.9 \mathrm{deg}{ }^{2}\left(0.23 \mathrm{deg}^{2}\right.$ each filter $)$ \\
Optical angular resolution & $0.04 " / \mathrm{pixel}(\mathrm{at} 0.8 \mu \mathrm{m})$ \\
IR angular resolution & $0.10 " / \mathrm{pixel}$ \\
Image stability & $0.03 "$ \\
Filters & Broad-band $(0.6-1 \mu \mathrm{m}), \mathrm{J}, \mathrm{H}, \& \mathrm{~K}(600-2500 \mathrm{~nm})$ \\
Optical detector & $2.6 \mathrm{k} \times 2.6 \mathrm{k} \mathrm{CCDs}$ of $10 \mu \mathrm{m} \mathrm{pixels}$ \\
IR detector & $2.3 \mathrm{k} \times 973 \mathrm{HgCdTe}$ devices of $18 \mu \mathrm{m}$ pixels \\
\hline
\end{tabular}

Table 2. Specifications of the X-ray system.

\begin{tabular}{ll}
\hline \hline Parameter & Value \\
\hline Focal length & $1600 \mathrm{~mm}$ \\
Diameter of one mirror system & $358 \mathrm{~mm}$ \\
Baffle length & $600 \mathrm{~mm}$ \\
Telescope size (diameter/length) & $0.4 \mathrm{~m} / 2.6 \mathrm{~m}$ \\
FOV & $0.473 \mathrm{deg}^{2}$ \\
Angular resolution & $<15^{\prime \prime}(\mathrm{at} 1 \mathrm{keV})$ \\
CCD size & $19.2 \times 19.2 \mathrm{~mm}^{2}$ \\
Pixel size & $75 \times 75 \mu \mathrm{m}^{2}$ \\
Readout time & $50 \mathrm{~ms}$ \\
Energy range & $0.5-10 \mathrm{keV}^{2}$ \\
Energy resolution & $130 \mathrm{eV} \mathrm{at} 6 \mathrm{keV}$ \\
Effective collecting area & $2656 \mathrm{~cm}^{2}$ \\
Total Grasp & $753.6 \mathrm{~cm}^{2} \mathrm{deg}^{2}($ at $1 \mathrm{keV})$ \\
\hline
\end{tabular}

\subsection{X-ray telescope and detectors}

The DEMON X-ray instrument consists of eight X-ray telescopes with a design similar to that developed for the eROSITA mission. Telescope specifications are explained in detail in the eROSITA Mission Definition Document (www.mpe.mpg.de/erosita/MDD-6.pdf).

Basically, each X-ray telescope is composed of 54 nested Wolter-I mirror shells (paraboloid+hyperboloid optics), with 27 outer shells to enhance the effective area at low energies. The optical design of the X-ray mirror system has hexagonal geometry, optimised to achieve maximum sensitivity between 0.5 and $10 \mathrm{keV}$. The mirrors are fabricated using a nickel-galvano plating, coated with gold to enhance their reflectivity. Each mirror module has its own CCD-detector, mounted in its own housing and equipped with its own electronics. The CCD size of $19.2 \times 19.2 \mathrm{~mm}^{2}$ corresponds to a FOV of $41.2^{\prime} \times 41.2^{\prime}$. The X-ray detectors are based on the pn-CCD principle, although they have a smaller pixel size $\left(75 \times 75 \mu \mathrm{m}^{2}\right)$ and shorter readout time than regular pn-CCDs. The semiconductor laboratory of MPE are already fabricating these kind of detectors. 
Table 3. Orbital parameters.

\begin{tabular}{ll}
\hline \hline Parameter & Value \\
\hline$\Theta_{\text {inclination }}$ & $5 \mathrm{deg}$ \\
Altitude & $600 \mathrm{~km}$ \\
Velocity & $7.6 \mathrm{~km} \mathrm{~s}^{-1}$ \\
Period & $1.61 \mathrm{~h}$ \\
$t_{\text {eclipse }}$ & $28 \mathrm{~min}$ \\
\hline
\end{tabular}

Table 4. Estimated masses and power requirements of the spacecraft components.

\begin{tabular}{lll}
\hline \hline Subsystem & Mass & Power \\
\hline Propellant & $500 \mathrm{~kg}$ & \\
Spacecraft structure \& Harness & $280 \mathrm{~kg}$ & \\
Telescope structural support & $160 \mathrm{~kg}$ & \\
Optical mirror system & $120 \mathrm{~kg}$ & \\
Optical/IR detectors & $200 \mathrm{~kg}$ & $480 \mathrm{~W}$ \\
X-ray telescope \& detectors & $700 \mathrm{~kg}$ & $110 \mathrm{~W}$ \\
Power system & $250 \mathrm{~kg}$ & $130 \mathrm{~W}$ \\
Thermal control \& Multi-layer insulation & $100 \mathrm{~kg}$ & $900 \mathrm{~W}$ \\
Attitude control & $90 \mathrm{~kg}$ & $250 \mathrm{~W}$ \\
Electronics \& Telemetry & $100 \mathrm{~kg}$ & $110 \mathrm{~W}$ \\
\hline Total & $\mathbf{2 5 0 0 ~ \mathbf { ~ k g }}$ & $\mathbf{1 9 8 0 ~ W}$ \\
\hline
\end{tabular}

\section{SPACECRAFT ORBIT AND SCANNING STRATEGY}

In order to achieve the scientific objectives of the mission and to meet the spacecraft's operational requirements, we have chosen a circular low-earth orbit (LEO) with the parameters given in Table 3. This particular orbit was chosen to avoid the Van Allen radiation belts and the South Atlantic Anomaly, both of which would prejudice X-ray observations. The selected LEO will also facilitate ground control and data download procedures. Because of atmospheric drag, periodical corrective maneuvers (carried out by thrusters) will be needed to reposition the spacecraft into its desired orbit. The propellant mass required for these maneuvers has been computed at $500 \mathrm{~kg}$ (for a 5 year mission). In total, the spacecraft's dry mass is $2000 \mathrm{~kg}$ ( $20 \%$ marging included), which fits well within the transport capabilities of $5500 \mathrm{~kg}$ of a Soyuz rocket launched from Kourou into the desired LEO orbit (see Table 4 for details).

The telescopes will be allowed to shift within a cone aligned with the Earth's rotational axis with a maximum semi-aperture angle $\theta_{\mathrm{sa}}=21 \mathrm{deg}$. This will avoid bringing the Earth, the Sun and the plane of the Milky Way into the instrumental FOV. The exact pointing range will also depend on the specific position of the Earth along its orbit. The selected value of $\theta_{\mathrm{sa}}$ allows, in principle, the coverage of about $15000 \mathrm{deg}^{2}$ of the sky around the Earth's north and south poles. The sky will be sampled in concentric circles while the center of each FOV will be shifted by $10^{\prime \prime}$ every two orbits in accordance with detector array technical requirements.

With an exposure time of $16000 \mathrm{~s}$ for each pointing, this configuration is expected to reach the scientific demands for a $5000 \mathrm{deg}^{2}$ survey in a 4.5 years mission (including $68 \%$ efficiency).

\section{SPACECRAFT ENGINEERING}

\subsection{Spacecraft design}

The DEMON spacecraft is divided in two main parts: the main body and the service module.

The main body is a $6 \mathrm{~m}$ high cylinder with $2.6 \mathrm{~m}$ of diameter. It contains the optical/IR and X-ray telescopes and detectors, the solar panels, and a radiator to cool the X-ray focal planes. To avoid any direct stray light into the mirror system, the optical/IR telescope will be protected by a cylindrical deployable baffle with a total 


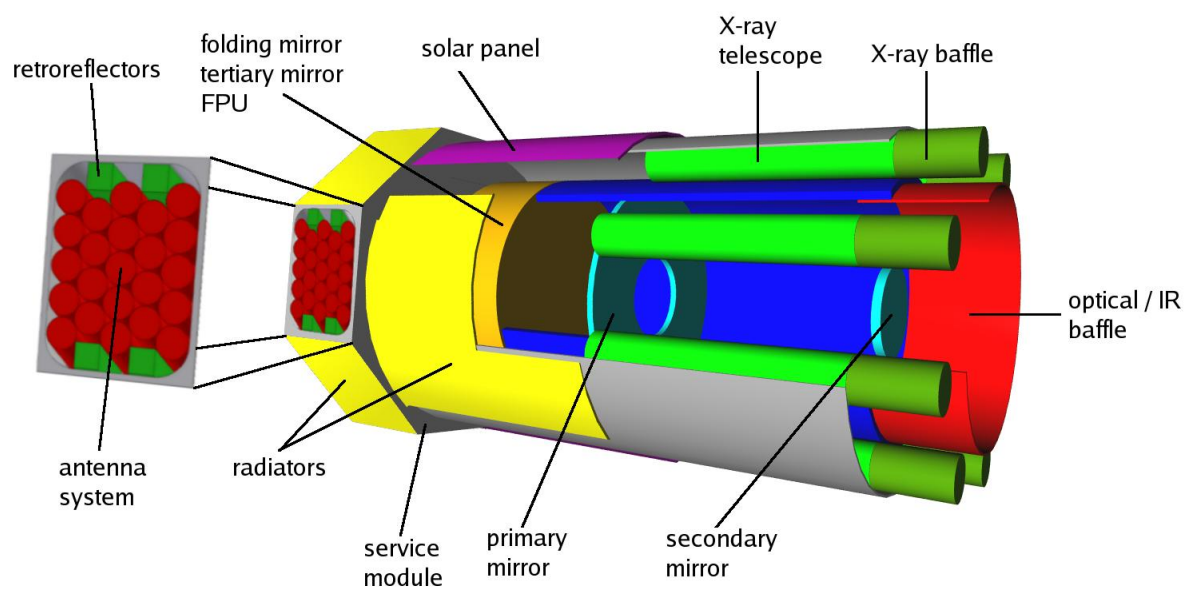

Figure 3. The design of the spacecraft.

Table 5. Estimated costs for a five years mission.

\begin{tabular}{ll}
\hline \hline Part & Cost \\
\hline Main telescope & $50 \mathrm{M} €$ \\
Optical/IR camera & $80 \mathrm{M} €$ \\
X-ray instrument & $16 \mathrm{M} €$ \\
Platform & $200 \mathrm{M} €$ \\
Launcher & $50 \mathrm{M} €$ \\
Ground support & $100 \mathrm{M} €(20 \mathrm{M} € / \mathrm{yr})$ \\
Ground observations & $12.5 \mathrm{M} €(2.5 \mathrm{M} € / \mathrm{yr})$ \\
\hline Total & $\mathbf{5 0 8 . 5} \mathbf{M}$ \\
\hline
\end{tabular}

length of $110 \mathrm{~cm}$ (70 $\mathrm{cm}$ outsize the main tube of the telescope). Each X-ray telescope has also its own cylindrical baffle with a total length of $60 \mathrm{~cm}$.

The service module is an octagonal box which is $1.1 \mathrm{~m}$ high and $3.75 \mathrm{~m}$ in diameter. The lateral sides include the phased array antenna system. The bottom side has two antennas, the Soyuz connection ring and radiators. At the sides of the service module there are two solar sensors, two star trackers and 12 thrusters. Inside the service module there is the thermal control system, the attitude control system, the power conditioning devices, the electronic devices, the telemetry and telecommand devices and the batteries.

An artist impression of the satellite is shown in Fig. 3.

\subsection{Power system}

During most of the orbit, the power of the spacecraft will be supplied by a solar array built from ultra triple junction solar cells of $\mathrm{GaInP}_{2}, \mathrm{GaAs}$, and Ge. The solar array will also charge batteries that will be used during the eclipses and in particular circumstances when greater amounts of power are needed. Lithium-ion batteries will be used since these have a large interval of allowed operational temperatures $(248 \mathrm{~K}-268 \mathrm{~K})$ facilitating thermal control.

The power needed for the different subsystems of the spacecraft are listed in Table 4. It amounts to a total required power of $1980 \mathrm{~W}$. The necessary size of the solar array is therefore $14.7 \mathrm{~m}^{2}$ taking into account the losses of energy during conversions, the cell efficiency and deterioration of components with time.

\subsection{Thermal control}

In order to minimize the radiative exchange and ensure the insulation, the satellite is wrapped with layers of aluminized mylar and covered with a special white paint. 
Due to instrumentation requirements, the service module and payload operates at different temperatures. For this reason they must be kept thermally insulated from each other. The service module is maintained at a temperature between $278 \mathrm{~K}$ and $303 \mathrm{~K}$ using a passive cooling system consisting of two radiators. The radiators are placed on the cold side of the service module to carry off the heat produced inside by the detector's electronic boards, attitude control, propellants, power and communication system and electronic internal dissipation. The batteries are thermally isolated and equipped with heaters controlled by thermostats. The two star trackers are cooled with Peltier coolers.

\subsubsection{Thermal control of the IR detector}

Active cooling is required for the IR detector since the focal plane has to be kept at $150 \mathrm{~K}$. For this purpose there are 16 thermoelectric pumps and 32 spares. Each pump has an absorption capacity of $1.22 \mathrm{~W}$ and a required power supply of $43.5 \mathrm{~W}$. A radiator is placed on the cold side of the spacecraft to radiate this power.

\subsubsection{Thermal control of the X-ray detector}

The focal plane of each X-ray detector has to be maintained at an operating temperature of $213 \mathrm{~K}$ in order to achieve optimal functionality and energy resolution. Since the adopted attitude strategy maintains one side of the spacecraft hidden from the sun and the earth (except for 1.5 minutes per orbit), passive cooling is a possible solution. The total heat flux coming from the albedo and IR radiation of the earth at that cold side was computed with the ESARAD software for different positions of the spacecraft along the orbit. Based on this result, we plan to use radiators and heat-pipes to cool the X-ray detectors. To avoid overheating the CCD focal plane during the hot cases along the orbit, active elements will support the cooling. Since the mirror system operate at ambient temperature, heaters with thermostats are mounted on the mirror modules in order to maintain temperature stability (e.g. $293 \pm 2 \mathrm{~K}$ ).

\subsection{Attitude control}

The thermal control requires two $180^{\circ}$ slews of the spacecraft every orbit: a direct slew, and a retrograde slew to return the spacecraft to its initial position (similar to the strategy adopted by eROSITA). Due to the short period of the orbit, the slew rate should be of the order $1^{\circ} / \mathrm{sec}$ (a quarter spin must be done in less than two minutes).

To accomplish the survey strategy, the spacecraft should be able to make slews in all three directions. Therefore, 4 momentum wheels (one for each axis) are used for the actuators. The spacecraft includes a spare momentum wheel in case one of the others breaks down. Twelve hydrazine thrusters are used for momentum dumping, orbital corrections and to assist in more demanding maneuvers, such as the $180^{\circ}$ slew.

To guarantee a high spacecraft stability, we have three different types of attitude control movements: maneuver, pointing and stability mode. For this purpose three types of sensors are used: two sun sensors, two star trackers, and two space inertia reference units. The maneuver mode consist of retrieving data from the two sun sensors and performing a fast slew. In the pointing mode, the star trackers permit more precise maneuvers. Finally, the stability mode is required for long time exposures when maximum stability is necessary. In this case, the space inertia reference unit (that uses the data from six gyroscopes) gives a precision of one arcsecond per hour. The use of a kilo-Hertz satellite laser ranging system as a backup has also been envisaged.

\subsection{Communication}

The DEMON communication, telemetry tracking and command (TT\&C) segment will be based on reliable radio frequency $(\mathrm{RF})$ technology in the Ka-band, since this is the only band which will be able to offer sufficient telemetry capacity. In a few years, the Ka-band technology will be fully space qualified and tested on several space-borne missions. The specifications of the memory and telecommunication subsystems are shown in Table 6 . 
Table 6. Parameters of the memory and telecommunications subsystem.

\begin{tabular}{ll}
\hline \hline Parameters, Hardware & Values, Annotations \\
\hline Frequency of the Ka-band TT\&C & 25.5-27 GHz (alternatively 37-38 GHz) \\
Bandwidth efficient modulations ${ }^{28}$ & OQPSK/PM, OQPSK I/Q, SOQPSK, FQPSK-B, etc. \\
Forward error correction codes & Turbo Code, Reed-Solomon; FPGA implementation \\
SSPAs & max. $1 \mathrm{~W}_{R F}$ each, in all $60 \mathrm{~W}_{D C}$ for power system \\
Telemetry data rate & $500 \mathrm{Msps}$ up to 1.2 Gsps (fair weather) \\
SSMM with lossless data compression & min. 500 GBit at end-of-life; on-board SAN \\
CCDs uncompressed raw data rate & max. continuous 160 Mbps \\
Data rate for SSMM / Compression factor & $60 \mathrm{Mbps} / 23$ \\
Ground stations & Kourou and Malindi, each with 500 s of visibility per orbit \\
\hline
\end{tabular}

\subsubsection{Space segment}

The baseline concept for the science and telemetry link are compact and efficient Ka-band solid-state power amplifiers (SSPAs), together with a "semi-active" conformal phased array antenna, Butler-like matrices, and MMIC phase shifters. ${ }^{25-27}$ The antenna has no moving part, as demanded for extremely fine pointing accuracy. The power transferred from the antenna system to the spacecraft is kept constant in order to minimize negative effects from a temperature change into the optical payload.

Science and engineering data are buffered in the solid-state mass memory (SSMM) on board, and loss-less compressed with a consultative committee for space data systems (CCSDS) compliant solution. This data is subsequently down-linked via the telemetry system and antenna to the ground station.

\subsubsection{Ground segment}

The orbit of DEMON enables the usage of ground stations with low cost, commercial Ka-band front-ends in Kourou/French Guiana and Malindi/Kenya. Beside the broadband telemetry link from the spacecraft to the ground stations, data relay system satellite services will be used with inter-orbit links (IOL) in Ka-band for telecommand and real-time engineering telemetry.

DEMON will also use several link management techniques for optimization, such as site-time diversity with selective re-transmission, short term forecast driven weather adaption, and error control coded operations.

\section{CONCLUSIONS}

This paper presents a new concept mission for a satellite aimed at provide better constraints on the cosmological parameters and, in particular, studying the evolution of the dark energy equation of state with time.

The innovate concept of the DEMON spacecraft is to carry out a combined optical/IR and X-ray survey of $5000 \operatorname{deg}^{2}$ to perform weak lensing and cluster statistics studies. The satellite will be complemented by one of the ground-based wide-field surveys currently under development.

For the optical/IR part of the survey, we investigated a baseline optical concept similar to the one proposed by SNAP. To satisfy the scientific requirements, the proposed telescope requires an additional magnification of 1.5 to sample the FWHM PSF with $2 \times 2$ pixels using CCDs of $10 \mu \mathrm{m}$. The derived consequences in the technical telescope design and image quality should be investigated in detail to determine the feasibility of this baseline.

For the X-ray part of the mission, we explore the possibility to include $8 \mathrm{X}$-ray telescopes in the spacecraft, taking advantage of the technology developed for the eROSITA mission. The result is an X-ray survey with a flux limit of $8 \times 10^{-15} \mathrm{erg} \mathrm{cm}^{-2} \mathrm{~s}^{-1}, 10$ times more sensitive than $\mathrm{DUO}^{29}$ and 5 times more sensitive than eROSITA.

Therefore, the DEMON 5000 $\mathrm{deg}^{2} \mathrm{X}$-ray survey is expected to discover 10 times more cluster per $\mathrm{deg}^{2}$ than DUO (with 6000deg ${ }^{2}$ survey) and even more clusters of galaxies than eROSITA (with an all-sky survey). With 
this amount of clusters we expect to obtain the best results so far from number density and power spectrum studies.

The final spacecraft is $7.1 \mathrm{~m}$ high and $3.75 \mathrm{~m}$ wide (at its broadest parts). It has an estimated mass of 2500 $\mathrm{kg}$, which is well within the transport capabilities of a Soyuz rocket into the desired LEO orbit. The estimated cost of the spacecraft is $510 \mathrm{M} €$ (see Table 5 for details).

Compared to SNAP, DEMON will perform a bigger survey and can be launched using a cheaper rocket. It also has the advantage of providing a proper sample of the FWHM PSF (and therefore better data quality for weak lensing studies), something which cannot be achieved by SNAP ${ }^{30}$ or JEDI. ${ }^{31}$ This, combined with the potential of the simultaneous X-ray survey, makes DEMON a very interesting concept design to be investigated for a future satellite to study dark matter and dark energy.

We also note that, although DEMON is optimized for a weak lensing survey, the high quality of the observations and the wide sky coverage of the survey will provide valuable data for other studies, like strong lensing systems, baryonic wiggles, galactic structure or detailed studies of clusters of galaxies. Due to the characteristics of the X-ray instruments, DEMON will also contribute to the study of the intracluster medium and galaxies with active nuclei (AGNs). Furthermore, a number of faint objects will be identified as future targets for narrow field instruments performing pointed imaging observations, like XEUS.

Finally, the planned far-infrared surveys based on the Sunyaev-Zel'dovich (SZ) effect (e.g. the ESA Planck mission and South Pole Telescope) will produce the redshift dependent cluster mass function and the power spectrum with different systematics compared to the DEMON X-ray survey, permitting for a very important cross-check of the two cosmological techniques to be made.

\section{ACKNOWLEDGMENTS}

The authors would like to thank the organizers and the tutors of the Alpbach summer school 2005 for inspiration and help. Especially they would like to thank Dr. Andreas Quirrenbach and the director of the school, Dr. Johannes Ortner. We would also like to thank Edo Loenen for help during the preparation of the 3D figure of the spacecraft, and the Space Research Institute of the Austrian Academy of Sciences and Dr. W. Baumjohann for the workshop support. A.B.A. would like to thank Dr. Jan Willem Pel and Dr. Isabel Pérez Martín for useful discussions. F.M. would like to thank CEA-DAPNIA/SAp for technical support and useful discussions during the preparation of the paper.

Financial support during the summer school, workshop and SPIE conference was obtained from ANGLES, ASI, CEA-DAPNIA/SPP, INFN of Bologna and Perugia, Danish Research Agency, DLR, Enterprise Ireland, Institute of Astrophysics University of Innsbruck, Austrian Research Promotion Agency FFG/ALR, GRICES, INAF, CIFS, NUI Maynooth Postgraduate Travel Fund, SNSB, SRON and Österreichische Forschungsförderungsgesellschaft mbH. The work by A.B.A. and M.Z. was financed by the European Community's Sixth Framework Marie Curie Research Training Network Programme under Contract No. MRTN-CT-2004-505183 ANGLES.

\section{REFERENCES}

1. Y. Mellier, "Probing the Universe with Weak Lensing," Ann. Rev. A\&A 37, pp. 127-189, 1999.

2. M. Bartelmann and P. Schneider, "Weak gravitational lensing," Physics Reports 340, pp. 291-472, 2001.

3. A. Refregier, "Weak Gravitational Lensing by Large-Scale Structure," Ann. Rev. A $\& A$ 41, pp. 645-668, 2003.

4. M. Jarvis, M. Takada, B. Jain, and G. Bernstein, "Weak Lensing Cosmology with LSST: Three-Point Shear Correlations," American Astronomical Society Meeting Abstracts 207, pp. -+, Dec. 2005.

5. Z. Haiman, S. Allen, N. Bahcall, and M. Bautz et al., "An X-ray Galaxy Cluster Survey for Investigations of Dark Energy," ArXiv Astrophysics e-prints , June 2005.

6. G. M. Voit, "Tracing cosmic evolution with clusters of galaxies," Reviews of Modern Physics 77, pp. 207-258, Apr. 2005.

7. T. H. Reiprich and H. Böhringer, "The Mass Function of an X-Ray Flux-limited Sample of Galaxy Clusters," ApJ 567, pp. 716-740, Mar. 2002. 
8. P. Rosati, S. Borgani, and C. Norman, "The Evolution of X-ray Clusters of Galaxies," Ann. Rev. A\&A 40, pp. 539-577, 2002.

9. S. Majumdar and J. J. Mohr, "Self-Calibration in Cluster Studies of Dark Energy: Combining the Cluster Redshift Distribution, the Power Spectrum, and Mass Measurements," ApJ 613, pp. 41-50, Sept. 2004.

10. K. Pedersen and H. Dahle, "Calibration of the Mass-Temperature Relation for Clusters of Galaxies Using Weak Gravitational Lensing," ArXiv Astrophysics e-prints , Mar. 2006.

11. A. Tyson, "LSST and Dark Energy," in ASP Conf. Ser. 339: Observing Dark Energy, pp. 95-+, Sept. 2005.

12. SNAP collaboration, "Supernova / Acceleration Probe: A Satellite Experiment to Study the Nature of the Dark Energy," ArXiv Astrophysics e-prints, May 2004.

13. C. Heymans, M. L. Brown, M. Barden, and J. A. R. Caldwell et al., "Cosmological weak lensing with the HST GEMS survey," MNRAS 361, pp. 160-176, July 2005.

14. P. Schneider, "Weak Gravitational Lensing," ArXiv Astrophysics e-prints, Sept. 2005.

15. T. Schrabback, J.-M. Miralles, T. Erben, and P. Schneider, "Cosmic Shear with ACS," in Baryons in Dark Matter Halos, Dec. 2004.

16. J. Rhodes, A. Refregier, N. R. Collins, and J. P. Gardner et al., "Measurement of Cosmic Shear with the Space Telescope Imaging Spectrograph," ApJ 605, pp. 29-36, Apr. 2004.

17. H. C. Ferguson, M. Dickinson, and M. Giavalisco et al., "The Size Evolution of High-Redshift Galaxies," ApJL 600, pp. L107-L110, Jan. 2004.

18. R. P. Kirshner, "Throwing Light on Dark Energy," Science 300, pp. 1914-1918, June 2003.

19. M. Lampton, M. Sholl, and SNAP Collaboration, "SNAP Telescope," American Astronomical Society Meeting Abstracts 207, pp. -+, Dec. 2005.

20. M. Bolzonella, J.-M. Miralles, and R. Pelló, "Photometric redshifts based on standard SED fitting procedures," $A \& A 363$, pp. 476-492, Nov. 2000.

21. C. R. Mullis, P. Rosati, G. Lamer, and H. Böhringer et al., "Discovery of an X-Ray-luminous Galaxy Cluster at $\mathrm{z}=1.4, " A p J L$ 623, pp. L85-L88, Apr. 2005.

22. D. Korsch, "Anastigmatic three-mirror telescope," Applied Optics 16, pp. 2074-2077, Aug. 1977.

23. J. M. Schuler, D. A. Scribner, and M. R. Kruer, "Alias reduction and resolution enhancement by a temporal accumulation of registered data from focal plane array sensors," in Proc. SPIE Vol. 4041, p. 94-102, Visual Information Processing IX, Stephen K. Park; Zia-ur Rahman; Eds., pp. 94-102, June 2000.

24. M. E. Levi and SNAP Collaboration, "GigaCAM: A One Billion Pixel Imager for the SNAP Satellite," Bulletin of the American Astronomical Society 32, pp. 1517-+, Dec. 2000.

25. E. Vourch, G. Caille, M. J. Martin, and J. R. Mosig et al., "Conformal Array Antenna for LEO Observation Platforms," IEEE Antennas and Propagation Society International Symposium 1, pp. 20-23, June 1998.

26. G. Caille, E. Vourch, M. J. Martin, and J. R. Mosig et al., "Conformal Array Antenna for Observation Platforms in Low Earth Orbit," IEEE Antennas and Propagation Magazine 44, pp. 103-104, June 2002.

27. A. M. Polegre, G. Caille, L. Boyer, and A. Roederer, "Semi-Active Conformal Array for ESA's GAIA Mission," IEEE Antennas and Propagation Society International Symposium 4, pp. 4108-4111, June 2004.

28. CCSDS, Bandwidth-efficient modulations, summary of definition, implementation, and performance, Consultative Committee for Space Data Systems, CCSDS 413.0-G-1, Green book, http://www.ccsds.org/, April 2003.

29. R. Griffiths, R. Petre, G. Hasinger, P. Predehl, and N. E. White et al., "DUO: the Dark Universe Observatory," in Proceedings of the SPIE, Volume 5488, pp. 209-221 (2004)., G. Hasinger and M. J. L. Turner, eds., pp. 209-221, Oct. 2004.

30. P. Marshall, R. Blandford, and M. Sako, "The SNAP strong lens survey [review article]," New Astronomy Review 49, pp. 387-391, Nov. 2005.

31. Y. Wang, A. Crotts, P. Garnavich, and W. Priedhorsky et al., "Joint Efficient Dark-energy Investigation (JEDI): a Candidate Implementation of the NASA-DOE Joint Dark Energy Mission (JDEM)," American Astronomical Society Meeting Abstracts 205, dec 2004. 